\title{
Estimating the Critical Duration for Temporal Summation of Standard Achromatic Perimetric Stimuli
}

Mulholland, P. J., Redmond, T., Garway-Heath, D. F., Zlatkova, M. B., \& Anderson, R. S. (2015). Estimating the Critical Duration for Temporal Summation of Standard Achromatic Perimetric Stimuli. Investigative Ophthalmology and Visual Science, 56, 431-437. https://doi.org/10.1167/iovs.14-15304

Link to publication record in Ulster University Research Portal

Published in:

Investigative Ophthalmology and Visual Science

Publication Status:

Published (in print/issue): 31/01/2015

DOI:

10.1167/iovs.14-15304

Document Version

Publisher's PDF, also known as Version of record

\section{General rights}

Copyright for the publications made accessible via Ulster University's Research Portal is retained by the author(s) and / or other copyright owners and it is a condition of accessing these publications that users recognise and abide by the legal requirements associated with these rights.

\section{Take down policy}

The Research Portal is Ulster University's institutional repository that provides access to Ulster's research outputs. Every effort has been made to ensure that content in the Research Portal does not infringe any person's rights, or applicable UK laws. If you discover content in the Research Portal that you believe breaches copyright or violates any law, please contact pure-support@ulster.ac.uk. 


\title{
Estimating the Critical Duration for Temporal Summation of Standard Achromatic Perimetric Stimuli
}

\author{
Pádraig J. Mulholland, ${ }^{1,2}$ Tony Redmond, ${ }^{3}$ David F. Garway-Heath, ${ }^{1}$ Margarita B. Zlatkova, ${ }^{2}$ \\ and Roger S. Anderson ${ }^{1,2}$
}

${ }^{1}$ National Institute for Health Research (NIHR) Biomedical Research Centre at Moorfields Eye Hospital NHS Foundation Trust and UCL Institute of Ophthalmology, London, United Kingdom

${ }^{2}$ Vision Science Research Group, School of Biomedical Sciences, University of Ulster, Coleraine, Northern Ireland, United Kingdom ${ }^{3}$ School of Optometry and Vision Sciences, Cardiff University, Cardiff, United Kingdom

Correspondence: Pádraig J. Mulholland, Moorfields Eye Hospital, 162 City Road, London, EC1V 2PD, UK; padraig.mulholland@moorfields.nhs. uk.

Submitted: July 23, 2014

Accepted: December 3, 2014

Citation: Mulholland PJ, Redmond T, Garway-Heath DF, Zlatkova MB, Anderson RS. Estimating the critical duration for temporal summation of standard achromatic perimetric stimuli. Invest Ophthalmol Vis Sci. 2015;56:431-437. DOI:10.1167/ iovs.14-15304
Purpose. To estimate the critical duration of temporal summation for achromatic Goldmann III stimuli under the conditions of standard automated perimetry (SAP) and quantify response variability for short-duration stimuli.

Methods. Contrast thresholds were gathered using the method of constant stimuli for seven circular $\left(0.48^{\circ}\right.$ diameter) incremental stimuli of varying duration (sum-of-frames equivalent: 8.3-198.3 ms), at an eccentricity of $8.8^{\circ}$ along the four principal meridians of the visual field in two healthy, psychophysically experienced observers. Stimuli were presented on a highresolution cathode ray tube display with a background luminance of $10 \mathrm{~cd} / \mathrm{m}^{2}$. Psychometric functions were fitted using a probit model and nonparametric local-linear analysis. The critical duration was estimated using iterative two-phase regression analysis, the results also being compared with values produced using previously published methods of analysis.

RESULTS. The median critical duration estimated using iterative two-phase regression analysis was $27.7 \mathrm{~ms}$ (IQR 22.5-29.8). A slight steepening of the psychometric function slope (lower variability) was observed for longer stimulus durations, using both probit and local-linear analysis techniques, but this was not statistically significant.

Conclusions. Critical duration estimates in this study are substantially shorter than those previously reported for a Goldmann III stimulus, under the conditions of SAP. Further work is required to firmly establish the relationship between measurement variability and the degree of local temporal and spatial summation.

Keywords: temporal summation, perimetry, measurement variability
S tudies of temporal summation have been reported in both $\checkmark$ basic $^{1-3}$ and clinical $^{4-6}$ psychophysics literature under a wide range of experimental conditions. Most studies have demonstrated the validity of Bloch's law, describing the reciprocal relationship between stimulus duration and threshold luminance, for a range of short-duration stimuli. The longest duration for which this relationship holds is termed the critical duration. Early reports of this parameter have been in the range of $100 \mathrm{~ms},{ }^{7}$ this varying with factors such as stimulus form (e.g., $\operatorname{area}^{8}$ ) and background luminance. ${ }^{2}$ It was with reference to such estimates that the durations of stimuli currently used in standard automated perimetry (SAP) were selected. ${ }^{9,10}$ It was proposed that durations between 100 and $200 \mathrm{~ms}$ are shorter than the latency of voluntary saccadic eye movements $(250 \mathrm{~ms})$ but longer than the upper limit of complete summation, or critical duration, making tests "less demanding for calibration." "The latter point makes the assumption that the critical duration and utilization time (duration at which the transition from partial to no summation occurs ${ }^{11}$ ) are coincidental. Thus, it was assumed that, for a stimulus of duration of $100 \mathrm{~ms}$ or more, contrast thresholds should be completely independent of duration (i.e., the summation curve is flat), necessitating that only the stimulus area and threshold contrast be specified. Despite such reasoning, the choice of stimulus duration is based on the presumed course of temporal summation for stimuli and test conditions that poorly resemble modern SAP. A reexamination of temporal summation for conventional SAP stimuli is, therefore, required.

A wide range of critical duration estimates have been reported between published studies. Such differences may be partially explained by different choices of stimulus area, ${ }^{8}$ background luminance, ${ }^{2}$ psychophysical task (detection or resolution), ${ }^{7,12}$ and possibly visual field locus. Of the published studies on this topic, only a small number have examined temporal summation for perimetric stimuli in healthy observers. Dannheim and Drance ${ }^{5}$ investigated the effect of stimulus duration on contrast thresholds for a $0.75^{\circ}$ achromatic stimulus at various visual field locations in both photopic $\left(3.18 \mathrm{~cd} / \mathrm{m}^{2}\right)$ and mesopic $\left(0.03 \mathrm{~cd} / \mathrm{m}^{2}\right)$ conditions. They estimated the critical duration to be approximately 100 and $320 \mathrm{~ms}$ in photopic and mesopic conditions, respectively, these values remaining constant across the visual field. Funkhouser and Fankhauser ${ }^{4}$ later examined temporal summation for a Goldmann III (GIII) stimulus under the conditions of SAP, in the fovea and at $14.1^{\circ}$ eccentricity, reporting critical duration values in the range of 52 to $78 \mathrm{~ms}$. Under identical conditions Okuyama and colleagues ${ }^{13}$ found the critical duration to be 
approximately $100 \mathrm{~ms}$, this remaining invariant of visual field locus between 0 and $26^{\circ}$ eccentricity. Considering these findings, it is evident that the photopic critical duration may not be constant at $100 \mathrm{~ms}$ as previously assumed, with estimates ranging from 52 to $100 \mathrm{~ms}$. Furthermore, in each of these studies, variable degrees of partial summation occur beyond the critical duration, indicating that the utilization time and critical duration may not be coincidental.

Critical duration values much shorter than $100 \mathrm{~ms}$ have been reported for test conditions and stimuli that closely resemble those used in SAP. Graham and Margaria ${ }^{8}$ reported the critical duration for a $0.26^{\circ}$-diameter stimulus to be approximately $30 \mathrm{~ms}$ at $15^{\circ}$ eccentricity under scotopic conditions. Owen ${ }^{14}$ found the critical duration for a $0.43^{\circ}$ stimulus to be approximately $50 \mathrm{~ms}$ for a background of 33.6 $\mathrm{cd} / \mathrm{m}^{2}$ and $100 \mathrm{~ms}$ in scotopic conditions. In these studies and others, ${ }^{2,15,16}$ it is also clear that the transition between complete and no summation is gradual, with little evidence for the critical duration and utilization time being coincidental. Significantly, this trend also is observed in those studies examining summation under the conditions of SAP, ${ }^{4,13}$ potentially leading to artificial overestimation of the critical duration as a result of the method used to analyze the data. For example, Okuyama et al. ${ }^{13}$ and Funkhouser and Fankhauser ${ }^{4}$ chose to fit linear regression lines of constrained slope, reflecting complete summation and no summation, respectively, to data points of short and long duration, the duration at which the extrapolated lines intersect being defined as the critical duration. This practice, although convenient, makes assumptions about the course of summation and fails to account for any partial summation. ${ }^{3}$ Considering this evidence, it may be hypothesized that (1) the true critical duration for a GIII stimulus under the conditions of SAP is shorter than the assumed $100 \mathrm{~ms}$, and (2) contrast thresholds for stimuli of duration of $100 \mathrm{~ms}$ or more are not independent of stimulus duration.

Critical duration estimates reported in the published literature also may be subject to imprecision secondary to high measurement variability for short-duration stimuli. ${ }^{17}$ It is well known that measurement variability for a standard achromatic GIII stimulus increases significantly in regions of the visual field where sensitivity is reduced in both healthy subjects and glaucoma patients. ${ }^{18,19}$ Variability also is known to increase for small area stimuli relative to large stimuli. ${ }^{20}$ If short-duration stimuli share such high measurement variability, the point in time at which summation changes from being complete to partial may be difficult to define, leading to unreliable estimates of the critical duration. To date, no study has quantified variability for SAP stimuli of short duration.

The aims of this study were to estimate the critical duration for a standard GIII stimulus under the conditions of SAP in healthy, psychophysically experienced observers, and quantify response variability for perimetric stimuli of short duration.

\section{MeTHODS}

\section{Subjects}

Two healthy experienced psychophysical observers were tested in this study: PJM ( 24 years) and RSA ( 47 years). Both had full visual fields as determined with a 24-2 SITA-Standard perimetry test (Humphrey Field Analyzer; Carl Zeiss Meditec, Dublin, CA, USA), normal IOP, and no detectable ocular disease. Best-corrected Snellen visual acuity was 6/5. Refractive error for subject RSA was +0.50 DS and for subject PJM -2.50 DS. Peripapillary retinal nerve fiber layer thickness, measured with a Spectralis OCT (Heidelberg Engineering, Heidelberg,
Germany) was within normal limits for both subjects. The right eye was used as the test eye for all experiments. All examinations were performed at the National Institute for Health Research Biomedical Research Centre based at Moorfields Eye Hospital and UCL Institute of Ophthalmology, London, UK. Ethical approval was gained from the LondonCentral National Research Ethics Service committee and the research protocol adhered to the tenets of the Declaration of Helsinki.

\section{Apparatus and Stimuli}

Stimuli were presented on a $\gamma$-corrected 21-inch Phillips FIMI MGD-403 achromatic monitor (Ampronix, Irvine, CA, USA), with a pixel resolution of $976 \times 1028$ and frame rate of $121 \mathrm{~Hz}$. All stimuli were generated using a ViSaGe MkII visual stimulus generator (Cambridge Research Systems, Rochester, UK) and Visual Psychophysics Engine software (v3.0; Cambridge Research Systems). A Cedrus RB-530 response pad (Cedrus Corporation, San Pedro, CA, USA) was used to collect participant responses. Stimuli were presented on an achromatic background of luminance $10 \mathrm{~cd} / \mathrm{m}^{2}$. The maximum luminance of test stimuli was $365 \mathrm{~cd} / \mathrm{m}^{2}$. Chromaticity coordinates of the background and stimuli were $x=0.258$ and $y=0.257$, as measured with a colorimeter (ColorCal-II; Cambridge Research Systems). Temporal summation was investigated by measuring contrast thresholds for GIII-equivalent achromatic stimuli (circular increments, $0.48^{\circ}$ diameter) with seven presentation times ranging from 8.3 to $198.3 \mathrm{~ms}$ (124 frames) when expressed as sum-of-frames equivalent. Four regions at $8.8^{\circ}$ eccentricity were examined along the $45^{\circ}, 135^{\circ}$, $225^{\circ}$, and $315^{\circ}$ meridians of the visual field. Subjects placed their head in a chin rest and rested their forehead against a bar. Refractive error was corrected for the viewing distance of 60 $\mathrm{cm}$ with full-aperture trial lenses.

\section{Psychophysical Procedure}

The method of constant stimuli was used to measure contrast thresholds for stimuli of varying duration. Thresholds were initially estimated at each test location for all stimulus durations using a randomly interleaved $1 / 1$ staircase with a Yes/No procedure. Each staircase terminated after six reversals, with the threshold estimate calculated as the mean of the final four reversals. Using these estimated threshold values, nine contrast levels were selected (four values above the estimated threshold, four values below it, and the estimated threshold itself) with an equal spacing of $0.05 \mathrm{log}$ units between each level. Twenty stimulus presentations were made at each contrast level and the proportion reported as "seen" recorded. Stimuli of varying contrast, test location, and duration were randomly interleaved, and presentations were limited to 630 per session with regular breaks provided. Measurements took place over 4 days with two sessions per day. Before each experiment, one drop of tropicamide hydrochloride $1 \%$ was instilled in the test eye to achieve a fixed pupil diameter. The pupil diameter was $8 \mathrm{~mm}$ for both subjects (retinal illuminance after correction for Stiles-Crawford effect $=247.3 \mathrm{td}$ ).

\section{Psychometric Function Fitting}

MATLAB (version R2011a; The MathWorks, Inc., Natick, MA, USA) and the model-free toolbox ${ }^{21}$ (version 1.1, available in the public domain at www.modelfree.manchester.ac.uk) were used to fit psychometric functions to the frequency-of-seeing data. Model-free uses a nonparametric local-linear analysis technique. This has the advantage of not assuming the true psychometric function to be described by a classic parametric model (e.g., 


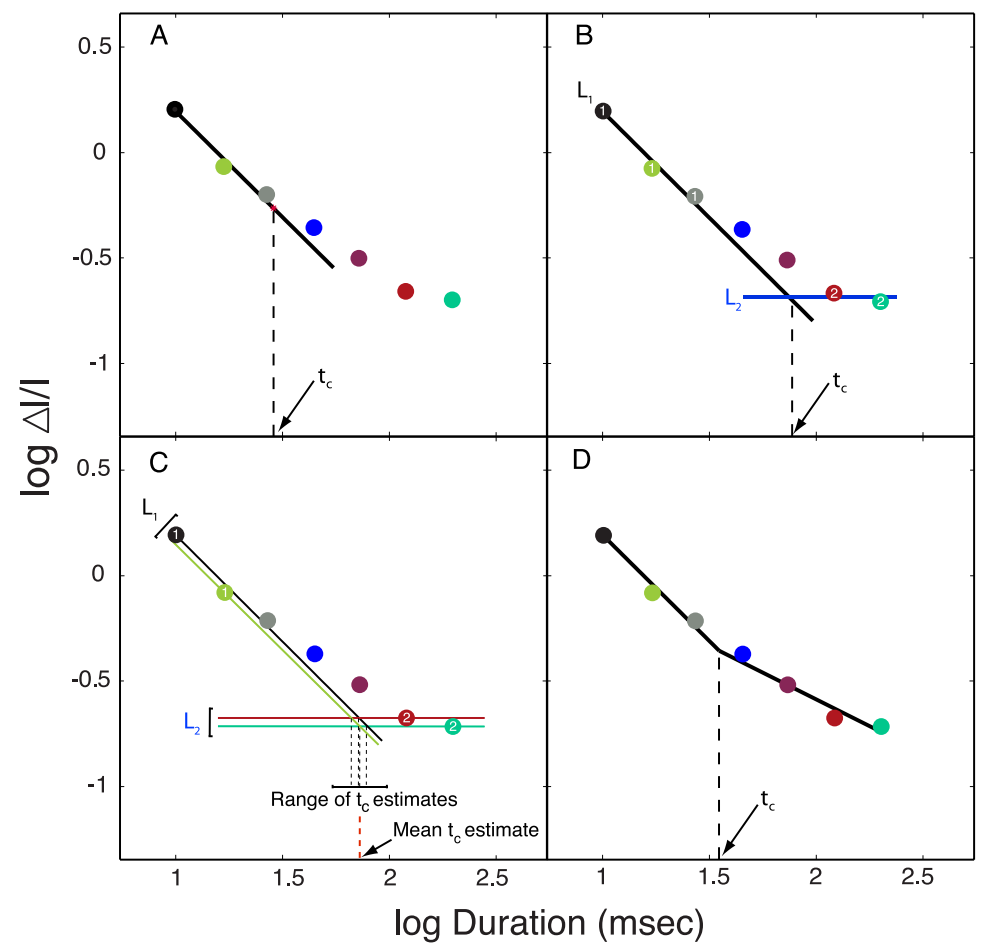

Figure 1. Methods used to estimate the critical duration $\left(\mathrm{t}_{\mathrm{C}}\right.$ ): (A) Manual estimation, (B) constrained least-squares regression analysis, (C) extrapolated intersection analysis, and (D) iterative two-phase regression analysis. See text and Supplementary Material for full description of each method.

cumulative Gaussian, logistic). The use of classic models, without knowledge of the true shape of the underlying function, can lead to large differences in estimated parameters, such as psychometric function slope and threshold. ${ }^{21}$ In our analysis, the guess and lapse rates were permitted to vary between $\mathrm{p}(0-0.1)$ and $\mathrm{p}(0.9-1.0)$, respectively, to prevent spurious estimates of slope, secondary to rates of false-positive and -negative responses that might otherwise be acceptable in clinical perimetry. ${ }^{22}$ Contrast thresholds were defined as the point on the psychometric function corresponding to $50 \%$ seen. Measurement variability for a given stimulus was represented by the slope of the psychometric function. For comparison, a generalized linear model (probit) was applied to the data using a constrained maximum likelihood method. ${ }^{22}$ Wilcoxon signed rank analysis was used to test for significant differences in the slope and threshold values obtained using the parametric (probit) and nonparametric (model-free) analysis.

\section{Estimating the Critical Duration}

To reflect the wide range of methods used to analyze summation data in the literature, we estimated the critical duration using those methods most commonly reported. Each method involves fitting two lines to the data (plot of log contrast thresholds against log stimulus duration). Differences between the methods lie in assumptions made about fixed or free parameters of each of the lines, the method by which remaining parameters are calculated, and how the critical duration is determined from the interaction between the two lines. For all analyses, a line of slope of -1 was used to describe complete summation (in accordance with Bloch's law) on a plot of log contrast threshold against log stimulus duration. Where required by the analysis method, a slope of zero was assumed for the slope of the summation function in a region where no summation is assumed to occur. The following methods were used to estimate the critical duration:

A. Manual estimation: This method required one of the authors (PJM) and an untrained analyst, naive to the aims of the study, to estimate, by eye, the point at which data points first deviate from a reference line with a slope of -1 . The critical duration for each summation function was defined as the mean of estimates for both analysts.

B. Constrained least-squares analysis ${ }^{23}$ : This analysis fits two lines of constrained slope (reflecting complete and no summation) to selected data points (in this study, the three shortest and two longest stimulus durations) using a least-squares method. The critical duration was estimated as the point at which the lines intersect.

C. Extrapolated intersection analysis ${ }^{4}$ : Like constrained least-squares analysis, this assumes that complete summation occurs for short-duration stimuli, with no summation for long-duration stimuli. Extrapolated intersections for four combinations of the shortest (8.3, $16.5)$ and longest $(124,198.3 \mathrm{~ms})$ stimulus durations were calculated, with the critical duration being taken as the mean of the four estimated values.

D. Iterative two-phase regression analysis ${ }^{24}$ : In this analysis, two lines were fitted to the data. The slope of the first line was constrained to -1 , to represent complete temporal summation. The slope and intercept of the second line were free to vary. The final position of the lines, including their intersection, was determined by least-squares analysis. This intersection (breakpoint) was taken to be the critical duration estimate.

Each method is described schematically in Figure 1 and in greater detail in the Supplementary Material. 


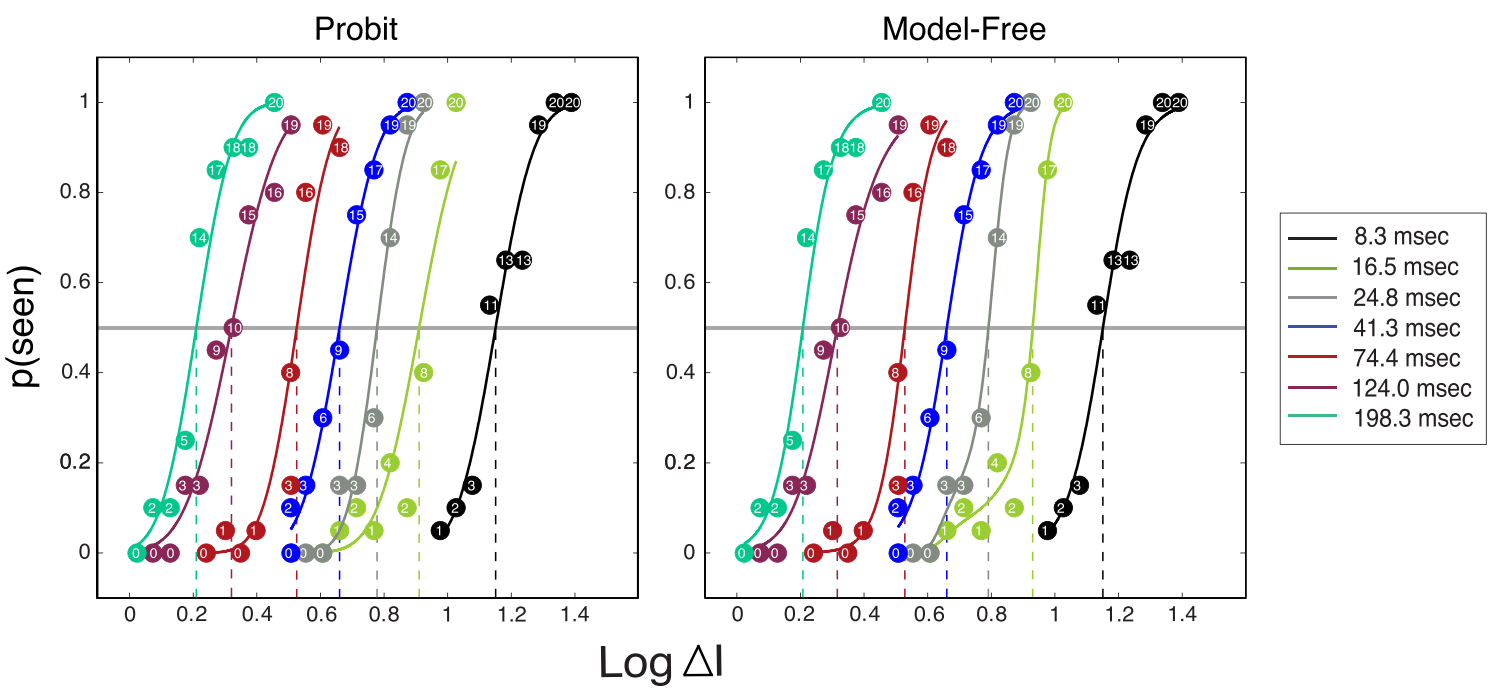

FIGURE 2. Example psychometric functions generated using probit and model-free methods for subject PJM. Data point labels indicate number of stimuli seen (of 20) and dashed lines to the abscissa the threshold. The $50 \%$ seen (p[seen] $=0.5$ ) level is highlighted on each plot with a gray line .

\section{RESULTS}

Example psychometric functions for subject PJM may be seen in Figure 2. There was no statistically significant difference in contrast thresholds, as estimated using probit or model-free methods for individual stimulus durations, with the exception of 10 and $27 \mathrm{~ms}$, where median contrast thresholds were 0.02 and $0.007 \mathrm{log}$ units higher, respectively, when calculated with the model-free method ( $P<0.05$, Wilcoxon signed rank test). Slope estimates were also significantly higher (i.e., psychometric function slope steeper) $(P<0.05)$ when calculated using model-free analysis for all stimulus durations, with the exception of 17,45 , and $121 \mathrm{~ms}(P>0.05)$. Box plots of slope values for both subjects, plotted as a function of stimulus duration, are shown in Figure 3. There was a slight flattening of psychometric functions with reduced stimulus duration over all locations in both subjects, with both probit and model-free fits, but this was not statistically significant $(P>0.05$, Friedman test).
Critical duration estimates generated using each analysis method are shown in Figure 4. In line with previous studies, ${ }^{4,13}$ those methods that constrain the slope of both lines in the summation function produce comparably longer critical duration values. The median critical duration values for the constrained least-squares $(63.2 \mathrm{~ms}$, interquartile range [IQR] 60.5-66.4) and extrapolated intersection analysis (62.2 ms, IQR 59.2-64.2) were significantly longer than both the manual estimation (36.7 ms, IQR 29.2-41.6) and two-phase regression (27.7 ms, IQR 22.5-29.8) methods (all $P<0.005$ ). The manual estimation method produced estimates that were significantly longer than two-phase regression analysis $(P<$ 0.05). There was no significant difference between the estimates produced using constrained least-squares and extrapolated intersection analysis $(P=0.38)$.

To examine whether contrast thresholds for a GIII stimulus are independent of stimulus duration when the critical duration is exceeded, a temporal summation function was plotted using median contrast thresholds across both subjects (Fig. 5). It can be clearly seen that beyond the critical duration

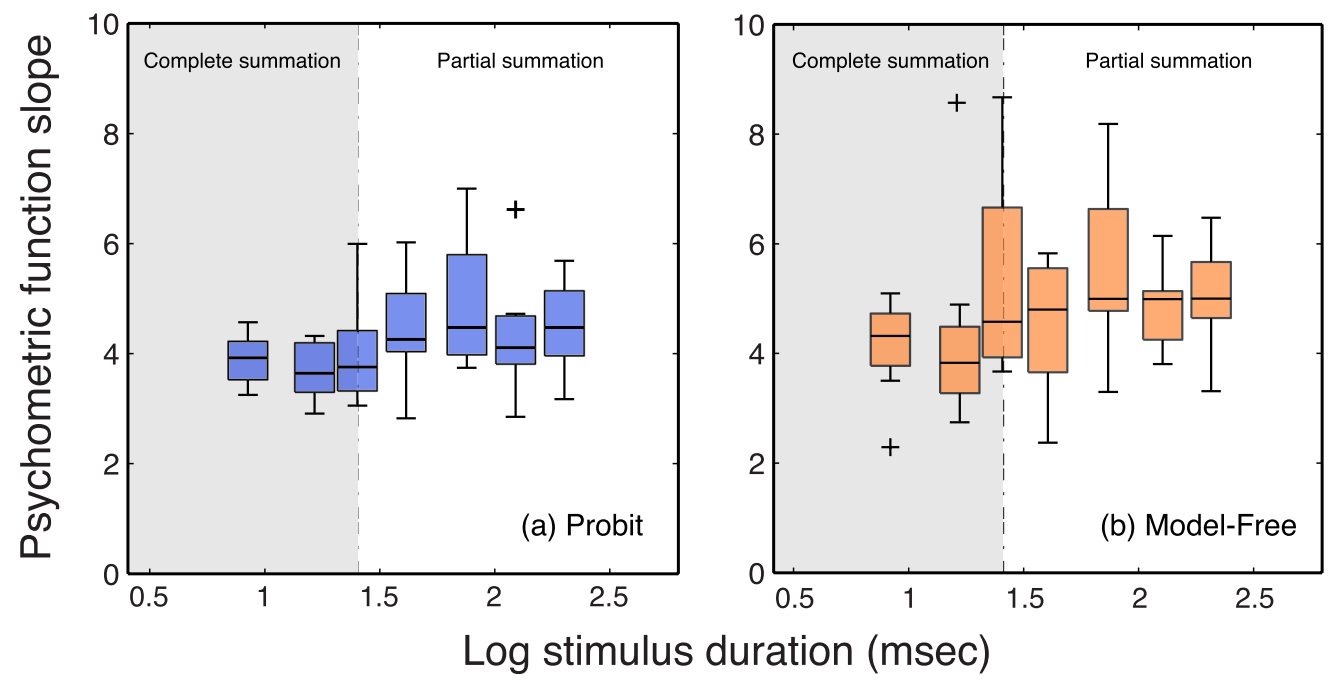

Figure 3. Psychometric function slopes for stimuli of varying duration. Values are those estimated using (a) probit and (b) model-free methods for both observers. Also included is the median critical duration estimate (dot-dashed line). 


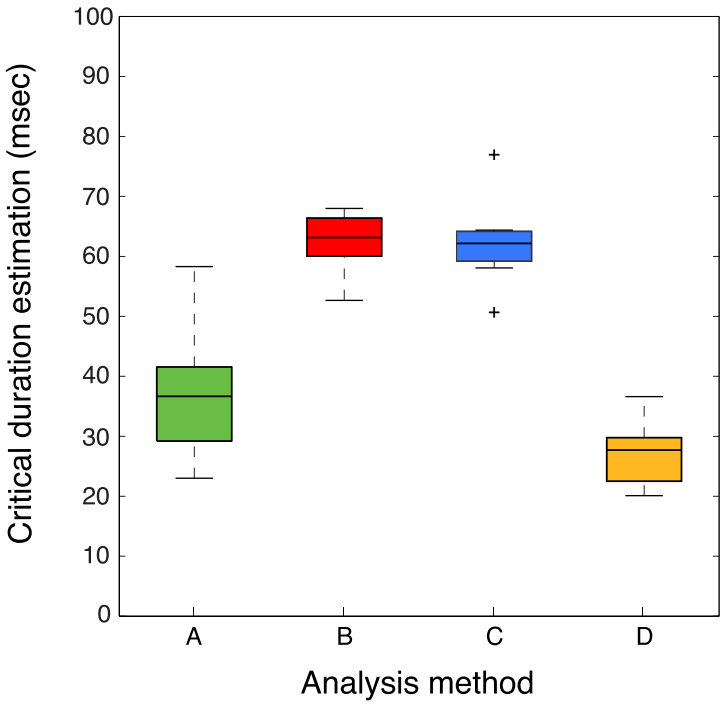

Figure 4. Critical duration estimates for all test locations generated using a variety of methods: (A) manual estimation, (B) constrained least-squares regression analysis, (C) extrapolated intersection analysis, and (D) iterative two-phase regression analysis.

(approximately $30 \mathrm{~ms}, 1.5 \mathrm{log} \mathrm{ms}$ ) contrast thresholds continue to decline (sensitivity continues to increase for longer stimulus durations). Further examination of the data also reveals contrast thresholds to be significantly higher for stimuli with durations in the range 30 to $100 \mathrm{~ms}(1.5-2 \log \mathrm{ms})$ when compared with thresholds for a $200 \mathrm{~ms}(2.3 \log \mathrm{ms})$ stimulus $(P<0.05$, Friedman test $)$.

\section{Discussion}

\section{Critical Duration for GIII Stimulus Under Conditions of SAP}

In this study, we examined the temporal summation characteristics for a standard, achromatic GIII stimulus under the conditions of SAP. We found the median critical duration to be $27.7 \mathrm{~ms}$ at $8.8^{\circ}$ eccentricity when estimated using iterative twophase regression analysis. This value is significantly shorter than those previously reported in the literature for the same stimulus, under similar test conditions. ${ }^{4,13}$ In this study, there were no instances in which summation reduces to the point where contrast thresholds become independent of duration (Fig. 5). Thus, we conclude that, under the conditions of this experiment, the critical duration and utilization time are not coincidental. This trend of a gradual transition between complete and no summation has been demonstrated in previous studies performed under similar test conditions. ${ }^{4,13}$

Methodological differences may explain the shorter critical duration values found in this study relative to earlier estimates. ${ }^{4,13}$ The first difference concerns the region of the visual field examined. Previous studies ${ }^{4,13}$ presented stimuli at eccentricities from 0 to $30^{\circ}$, with critical duration estimates ranging from 52 to $100 \mathrm{~ms}$. Despite these differences, the effect of visual field eccentricity on temporal summation has been reported to be negligible when investigated using a constant stimulus diameter (Mulholland $\mathrm{P}$, et al. IOVS 2013;54:ARVO E-Abstract 3924). ${ }^{5,13}$ Furthermore, Okuyama et al. ${ }^{13}$ found the critical duration at $8^{\circ}$ along the $135^{\circ}$ meridian to be approximately $100 \mathrm{~ms}$ under identical conditions to this study. The second methodological difference relates to the analysis used to estimate the critical duration. Previous studies

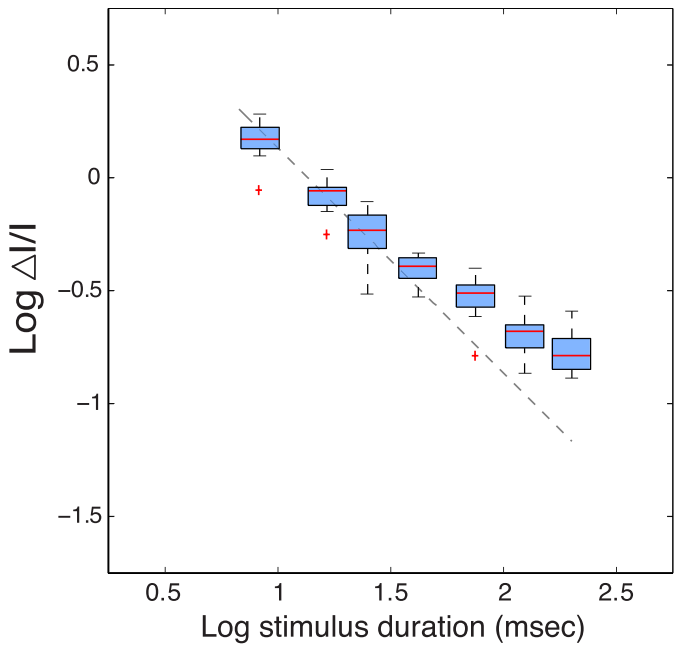

Figure 5. Contrast thresholds determined from probit fits for each stimulus duration examined. A dashed line of slope -1 (placed by eye) is included as reference to the trend expected when summation is complete.

have used analysis techniques that assume complete and no summation to be exhibited in the same data set (e.g., constrained least-squares regression ${ }^{23}$ ), whereas iterative twophase regression analysis, ${ }^{24}$ as used in this study, assumes only complete summation, with a variable degree of partial summation. As complete or partial summation was evident for all stimulus durations in this study, those methods that constrain the slope of both lines in the summation function introduce assumptions that do not respect normal physiology, and can lead to considerable inaccuracies when estimating the critical duration. This may be seen in the sample summation function in Figure 6. In this example, iterative two-phase regression analysis (solid line) produces a comparatively shorter critical duration when compared with constrained least-squares analysis (dashed line), the latter analysis method designating regions of partial summation to be complete.

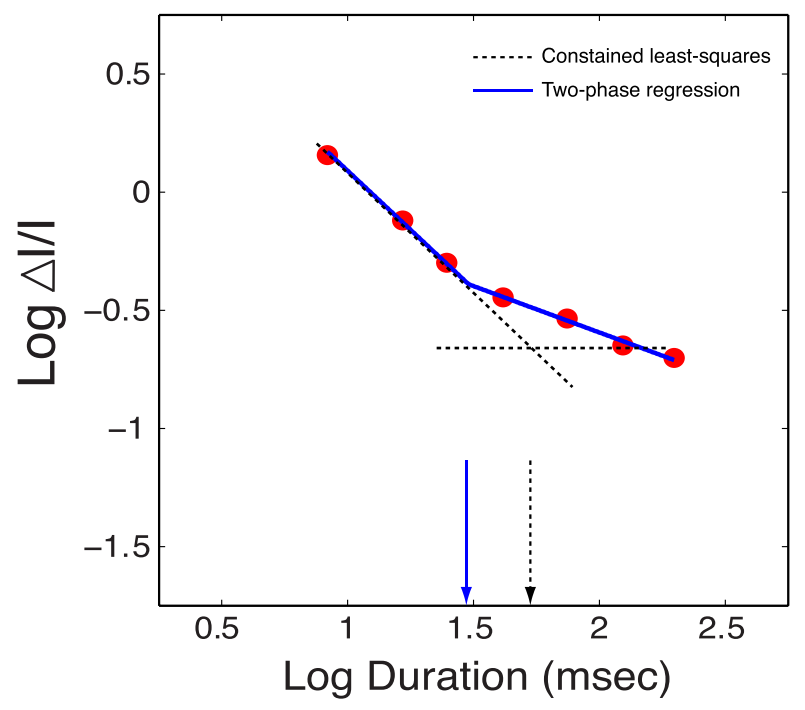

Figure 6. Comparison of critical duration estimates generated using iterative two-phase regression analysis (blue line) and constrained least-squares regression analysis (black dashed line) for data collected along the $315^{\circ}$ meridian in subject PJM. Summation functions predicted using each method are included for reference. 
Indeed, when the data in this study were analyzed using the methods proposed by previous studies, comparable critical duration estimates to those in the literature were produced (Fig. 4). As the exact nature of partial summation, and how it varies with stimulus duration and other experimental conditions is far from clear, ${ }^{8,16}$ it would seem that a nonlinear regression technique in which only the first component line is constrained to a slope of -1 (to reflect Bloch's law), might currently be the most appropriate analysis technique for the analysis of temporal summation data.

\section{SAP Stimulus Duration}

The findings of this study are somewhat at odds with the rationale underlying the earlier recommendation of presentation durations in the range 100 to $200 \mathrm{~ms}$ for SAP. ${ }^{9}$ This selection was made based on published reports that the critical duration is approximately $100 \mathrm{~ms}$. Such estimates are, however, likely the result of inappropriate analysis techniques, in addition to variations in stimulus characteristics, such as area and chromaticity, used in previous studies. It is also highly unlikely that contrast thresholds are independent of stimulus duration for conventional SAP stimuli with presentation durations in the range 100 to $200 \mathrm{~ms}$. The decision to use stimulus durations in this range also was made in the absence of knowledge of how temporal summation might change with diseases of the visual pathway, or with visual field eccentricity, particularly given its association with spatial summation, which has been shown to be altered in early glaucoma. ${ }^{25,26}$ For example, if temporal summation were also altered in glaucoma, it is possible that the sensitivity of SAP to disease is inhibited by using a stimulus duration in the range of 100 to $200 \mathrm{~ms}$. Clearly, there is a need to reexamine the choice of stimulus duration for use in SAP, if the aim of the test is to reliably uncover subtle changes in visual physiology in early disease.

\section{Measurement Variability for Stimuli of Short Duration}

The source of response variability for perimetric stimuli is a matter of debate within the literature. Previous studies have suggested this to be linked to retinal ganglion cell (RGC) density underlying the stimulus. Such arguments are founded on the observation that variability increases in regions of the visual field with reduced sensitivity in both healthy and glaucomatous eyes. ${ }^{18,19}$ A number of studies also have reported variability to be reduced for stimuli of large diameter (Gardiner S, et al. IOVS 2013;54:ARVO E-Abstract 2636 and Refs. 27, 28). In both healthy and glaucomatous observers, threshold variability increases when sensitivity is lower and brighter stimuli are needed to reach threshold. ${ }^{18,19,29,30}$ Because brighter stimuli are needed to reach threshold for shorter-duration stimuli, one might expect greater variability with short-duration stimuli. In this study, we observed psychometric functions to be very slightly steeper (less variable measurements) for stimuli of longer duration. This difference, however, failed to reach statistical significance for slopes estimated using both probit and model-free analysis.

Considering the findings of previous studies, in addition to those found in this study, it may be suggested that measurement variability for SAP stimuli is, in part, governed by the relationship between the underlying RGC density and the energy required at threshold at a given visual field locus. A similar theory was proposed by Redmond et al. ${ }^{25}$ to explain differences in test-retest variability with Goldmann size III and $\mathrm{V}$ stimuli in a group of glaucoma patients. In the case of increased measurement variability in damaged regions of the visual field in glaucoma, similar reasoning may be applied. If changes in sensitivity to a GIII stimulus in early glaucoma can be accounted for by an enlargement of Ricco's area, ${ }^{25}$ it is possible that changes in variability with increasing threshold for a fixed stimulus area also relate to changes in spatial summation. Verdon-Roe et al. ${ }^{31}$ also refer to stimulus energy as the primary determinant of psychometric function slope when determining thresholds in a motion-displacement hyperacuity test. Further work is, however, required to explicitly examine the relationship between stimulus energy, RGC density, and the degree of summation exhibited for a given stimulus. In this study, only two participants were included, together with only two stimulus durations below the critical duration, making it difficult to firmly relate the degree of summation to threshold variability, as well as limiting the power of any statistical tests performed. From Figure 3 it also may be seen that there is considerable variation in the psychometric function slope values for each stimulus duration used within the study.

\section{Conclusions}

The critical duration for a standard, achromatic GIII stimulus is approximately $30 \mathrm{~ms}$ when estimated using iterative two-phase regression analysis. This value is much shorter than those previously reported in published literature. Such discrepancies likely lie in the use of analysis techniques that make inappropriate assumptions about the degree of partial summation at differing locations, under varying experimental conditions. Further work is required to gain an insight into the association between the level of summation and variability in disease, in order to guide the selection of more appropriate perimetric stimuli for use in SAP.

\section{Acknowledgments}

Presented in part at the British Congress of Optometry and Vision Science annual meeting, Bradford, United Kingdom, in 2012.

Supported by a PhD studentship from the Department for Employment and Learning, Northern Ireland (PJM), and in part by the National Institute for Health Research Biomedical Research Centre based at Moorfields Eye Hospital NHS Foundation Trust and UCL Institute of Ophthalmology (PJM, DFG-H). DFG-H's chair at UCL is supported by funding from the International Glaucoma Association. The views expressed are those of the authors and not necessarily those of the NHS, the NIHR, or the Department of Health.

Disclosure: P.J. Mulholland, None; T. Redmond, Heidelberg Engineering (F); D.F. Garway-Heath, Carl Zeiss Meditec (F R), Heidelberg Engineering (F, R), P; M.B. Zlatkova, None; R.S. Anderson, Heidelberg Engineering (F, R)

\section{References}

1. McDougall W. The variation of the intensity of visual sensation with the duration of the stimulus. Br J Psychol. 1904;1:151189.

2. Barlow HB. Temporal and spatial summation in human vision at different background intensities. J Pbysiol (Lond). 1958; 141:337-350.

3. Watson AB. Temporal sensitivity. In: Boff KR, Kaufman L, Thomas JP, eds. Handbook of Perception and Human Performance. New York: Wiley; 1986:6.1-6.43.

4. Funkhouser AT, Fankhauser F. Temporal summation measurements with the Octopus 1-2-3 perimeter. Ger J Opbthalmol. 1994;3:120-128.

5. Dannheim F, Drance S. Studies of temporal summation of central retinal areas in normal people of all ages. Opbthalmol Res. 1971;2:295-303. 
6. Dannheim F, Drance S. Psychovisual disturbances in glaucoma: a study of temporal and spatial summation. Arch Ophthalmol. 1974;91:463-468.

7. Bruder G, Kietzman M. Visual temporal integration for threshold, signal detectability, and reaction time measures. Atten Percept Psychophys. 1973;13:293-300.

8. Graham C, Margaria R. Area and the intensity-time relation in the peripheral retina. Am J Physiol. 1935;113:299-305.

9. International Council of Ophthalmology. Perimetric Standards and Perimetric Glossary of the International Council of Ophthalmology. The Hague: Dr W. Junk bv Publishers; 1979.

10. Henson DB. Visual Fields. 2nd ed. Oxford: ButterworthHeinemann; 2000

11. Kietzman M, Gillam B. Visual temporal integration and simple reaction time. Atten Percept Psychophys. 1972;11:333-340.

12. Graham C, Cook C. Visual acuity as a function of intensity and exposure-time. Am J Psychol. 1937;49:654-661.

13. Okuyama S, Matsumoto C, Uyama K, Otsuiand O, Otori T. The influence of the stimulus duration on perimetric thresholds in the central $30^{\circ}$ of the visual field. In: Mills RP, Wall M, eds. Perimetry Update 1994/95 Proceedings of the XIth International Perimetric Society Meeting. New York: Kugler; 1995: 241-248.

14. Owen W. Spatio-temporal integration in the human peripheral retina. Vision Res. 1972;12:1011-1026.

15. Baumgardt E, Hillmann B. Duration and size as determinants of peripheral retinal response. J Opt Soc Am A. 1961;51:340-344.

16. Baumgardt E. Visual spatial and temporal summation. Nature. 1959;184:1951-1952.

17. Krakau C. Temporal summation and perimetry. Ophthalmic Res. 1989;21:49-55.

18. Chauhan B, Tompkins JD, LeBlanc RP, McCormick TA. Characteristics of frequency-of-seeing curves in normal subjects, patients with suspected glaucoma, and patients with glaucoma. Invest Ophthalmol Vis Sci. 1993;34:3534-3540.

19. Henson DB, Chaudry S, Artes PH, Faragher EB, Ansons A. Response variability in the visual field: comparison of optic neuritis, glaucoma, ocular hypertension, and normal eyes. Invest Ophthalmol Vis Sci. 2000;41:417-421.
20. Gilpin LB, Stewart WC, Hunt HH, Broom CD. Threshold variability using different Goldmann stimulus sizes. Acta Ophthalmol (Copenh). 1990;68:674-676.

21. Zychaluk K, Foster DH. Model-free estimation of the psychometric function. Atten Percept Psychophys. 2009;71:14141425.

22. Wichmann FA, Hill NJ. The psychometric function: I. Fitting, sampling, and goodness of fit. Percept Psychophys. 2001;63: 1293-1313.

23. Krauskopf J, Mollon JD. The independence of the temporal integration properties of individual chromatic mechanisms in the human eye. J Physiol. 1971;219:611-623.

24. Seber GAF, Wild CJ. Nonlinear Regression. New York: John Wiley \& Sons; 1989.

25. Redmond T, Garway-Heath DF, Zlatkova MB, Anderson RS. Sensitivity loss in early glaucoma can be mapped to an enlargement of the area of complete spatial summation. Invest Ophthalmol Vis Sci. 2010;51:6540-6548.

26. Wilson ME. Spatial and temporal summation in impaired regions of the visual field. J Physiol. 1967;189:189-208.

27. Wall M, Kutzko KE, Chauhan BC. Variability in patients with glaucomatous visual field damage is reduced using size V stimuli. Invest Ophthalmol Vis Sci. 1997;38:426-435.

28. Wall M, Doyle CK, Zamba KD, Artes P, Johnson CA. The repeatability of mean defect with size III and size V standard automated perimetry. Invest Ophthalmol Vis Sci. 2013;54: 1345-1351.

29. Artes PH, Hutchison DM, Nicolela MT, LeBlanc RP, Chauhan BC. Threshold and variability properties of matrix frequencydoubling technology and standard automated perimetry in glaucoma. Invest Ophthalmol Vis Sci. 2005;46:2451-2457.

30. Russell RA, Crabb DP, Malik R, Garway-Heath DF. The relationship between variability and sensitivity in large-scale longitudinal visual field data. Invest Ophthalmol Vis Sci. 2012; 53:5985-5990.

31. Verdon-Roe GM, Westcott MC, Viswanathan AC, Fitzke FW, Garway-Heath DF. Exploration of the psychophysics of a motion displacement hyperacuity stimulus. Invest Ophthalmol Vis Sci. 2006;47:4847-4855. 